FACTA UNIVERSITATIS (NIŠ)

Ser. Math. Inform. Vol. 35, No 5 (2020), 1273-1290

https://doi.org/10.22190/FUMI2005273C

\title{
ON THE FIXED-CIRCLE PROBLEM
}

\author{
Ufuk Çelik and Nihal Özgür
}

(C) 2020 by University of Niš, Serbia | Creative Commons Licence: CC BY-NC-ND

Abstract. In this paper, we focus on the geometric properties of fixed-points of a selfmapping and obtain new solutions to a recent problem called "fixed-circle problem" in the setting of an $S$-metric space. For this purpose, we develop various techniques by defining new contractive conditions and using some auxiliary functions. Furthermore, we present new examples to support our theoretical results.

Keywords: fixed-points; $S$-metric space; self-mapping.

\section{Introduction}

It is known that the fixed-point theory has been generalized by various approaches. One of these approaches is to generalize the used contractive condition (for example see [2], [5]). The other is to generalize the used metric space (see $[1,8,21,23]$ and the references therein). For example, in [21], Sedghi, Shobe and Aliouche presented the notion of an $S$-metric space as the generalization of a metric space. Then, some fixed-point theorems have been extensively studied on $S$-metric spaces (see $[6,7,9,13,15,18,19,21,22,24,25,27]$ for more details).

On the other hand, fixed-point theorems have been widely studied with different aspects such as the uniqueness of a fixed-point, common fixed point, etc. If a fixed point is not unique then the investigation of the geometric properties of fixed points of a self-mapping is an interesting problem. As a recent approach, the concept of a fixed circle and the fixed-circle problem have been presented on a metric (resp. an $S$-metric) space as a new direction of the generalization of known fixed-point results (see [17] and [16]). Then, new fixed circle theorems have been given by various techniques on metric (resp. $S$-metric) spaces (see $[11,12,20,26]$ for the metric case; $[10,14,24,25]$ for the $S$-metric case).

Our aim in this paper is to obtain new fixed-circle theorems for self-mappings on an $S$-metric space. In Section 2., we recall some basic facts about $S$-metric spaces.

Received October 31, 2019; accepted January 05, 2020

2020 Mathematics Subject Classification. Primary 47H10; Secondary 47H09, 54H25 
In Section 3., we give new fixed-circle theorems by introducing new types of the notion of an $F_{c}^{S}$-contraction introduced and used in [10]. In Section 4., we investigate new existence and uniqueness theorems for fixed circles of self-mappings using some auxiliary functions and contractive conditions. We support our theoretical results by illustrative examples.

\section{Preliminaries}

In this section, we recall some necessary notions and results on $S$-metric spaces with new examples.

Definition 2.1. [21] Let $X$ be a nonempty set and $\mathcal{S}: X^{3} \rightarrow[0, \infty)$ be a function satisfying the following conditions for all $x, y, z, a \in X$ :

1. $\mathcal{S}(x, y, z)=0$ if and only if $x=y=z$,

2. $\mathcal{S}(x, y, z) \leq \mathcal{S}(x, x, a)+\mathcal{S}(y, y, a)+\mathcal{S}(z, z, a)$.

Then $\mathcal{S}$ is called an $S$-metric on $X$ and the pair $(X, \mathcal{S})$ is called an $S$-metric space.

Example 2.1. [21] Let $X=\mathbb{R}$ (or $\mathbb{C}$ ) and the function $\mathcal{S}: X^{3} \rightarrow[0, \infty)$ be defined by

$$
\mathcal{S}(x, y, z)=|x-z|+|y-z|,
$$

for all $x, y, z \in \mathbb{R}\left(\right.$ or $\mathbb{C}$ ). Then the function $\mathcal{S}: X^{3} \rightarrow[0, \infty)$ is an $S$-metric and it is called the usual $S$-metric on $\mathbb{R}$ (or $\mathbb{C}$ ).

Lemma 2.1. [21] Let $(X, \mathcal{S})$ be an $S$-metric space and $x, y \in X$. Then we have

$$
\mathcal{S}(x, x, y)=\mathcal{S}(y, y, x) .
$$

It was given the relationships between a metric and an $S$-metric in the following lemma [7].

Lemma 2.2. [7] Let $(X, d)$ be a metric space. Then the following properties are satisfied:

1. $\mathcal{S}_{d}(x, y, z)=d(x, z)+d(y, z)$ for all $x, y, z \in X$ is an $S$-metric on $X$.

2. $x_{n} \rightarrow x$ in $(X, d)$ if and only if $x_{n} \rightarrow x$ in $\left(X, \mathcal{S}_{d}\right)$.

3. $\left\{x_{n}\right\}$ is Cauchy in $(X, d)$ if and only if $\left\{x_{n}\right\}$ is Cauchy in $\left(X, \mathcal{S}_{d}\right)$.

4. $(X, d)$ is complete if and only if $\left(X, \mathcal{S}_{d}\right)$ is complete.

The metric $\mathcal{S}_{d}$ was called as the $S$-metric generated by $d$ in [13].

Now we give a new example of an $S$-metric generated by a metric. 
Example 2.2. Let $X \neq \varnothing, d: X^{2} \rightarrow[0, \infty)$ be any metric on $X$ and the function $\mathcal{S}: X^{3} \rightarrow[0, \infty)$ be defined by

$$
\mathcal{S}(x, y, z)=\min \{1, d(x, z)\}+\min \{1, d(y, z)\} .
$$

Then the function $\mathcal{S}: X^{3} \rightarrow[0, \infty)$ is an $S$-metric on $X$ and the pair $(X, \mathcal{S})$ is an $S$ metric space. Clearly, this $S$-metric $\mathcal{S}$ is generated by the metric $m$ defined as $m(x, y)=$ $\min \{1, d(x, y)\}$.

There are some examples of an $S$-metric which is not generated by any metric (see [7], [10], [14] and [13]). We give a new example.

Example 2.3. Let $X=\mathbb{R}, d: X^{2} \rightarrow[0, \infty)$ be any metric on $X$ and the function $\mathcal{S}: X^{3} \rightarrow[0, \infty)$ be defined by

$$
\mathcal{S}(x, y, z)=\min \{1, d(x, z)\}+|y-z| .
$$

Then the function $\mathcal{S}: X^{3} \rightarrow[0, \infty)$ is an $S$-metric on $X$ which is not generated by any metric and the pair $(X, \mathcal{S})$ is an $S$-metric space. Conversely, assume that there exists a metric $d_{1}$ such that

$$
\mathcal{S}(x, y, z)=d_{1}(x, z)+d_{1}(y, z)
$$

for all $x, y, z \in X$. Then we obtain

$$
\mathcal{S}(x, x, z)=2 d_{1}(x, z) \Rightarrow d_{1}(x, z)=\frac{1}{2} \min \{1, d(x, z)\}+\frac{1}{2}|x-z|
$$

and

$$
\mathcal{S}(y, y, z)=2 d_{1}(y, z) \Rightarrow d_{1}(y, z)=\frac{1}{2} \min \{1, d(y, z)\}+\frac{1}{2}|y-z|,
$$

for all $x, y, z \in X$. So we get

$$
\begin{gathered}
\min \{1, d(x, z)\}+|y-z| \neq \frac{1}{2} \min \{1, d(x, z)\}+\frac{1}{2}|x-z| \\
+\frac{1}{2} \min \{1, d(y, z)\}+\frac{1}{2}|y-z|,
\end{gathered}
$$

which is a contradiction. Hence $\mathcal{S}$ is not generated by any metric.

Definition 2.2. [16] Let $(X, \mathcal{S})$ be an $S$-metric space. Then a circle and a disc are defined on an $S$-metric space as follows, respectively:

$$
C_{x_{0}, r}^{S}=\left\{x \in X: \mathcal{S}\left(x, x, x_{0}\right)=r\right\}
$$

and

$$
D_{x_{0}, r}^{S}=\left\{x \in X: \mathcal{S}\left(x, x, x_{0}\right) \leq r\right\}
$$

Example 2.4. Let $X$ be a nonempty set, the function $d: X^{2} \rightarrow[0, \infty)$ be any metric on $X$ and the $S$-metric space $(X, \mathcal{S})$ be defined as in Example 2.2. Let us consider the circle $C_{x_{0}, r}^{S}$ according to the $S$-metric $\mathcal{S}$ :

$$
C_{x_{0}, r}^{S}=\left\{x \in X: \mathcal{S}\left(x, x, x_{0}\right)=2 \min \left\{1, d\left(x, x_{0}\right)\right\}=r\right\} .
$$


Then we have the following cases:

Case $1:$ If $r=2$ then $C_{x_{0}, r}^{S}=\left\{x \in X: d\left(x, x_{0}\right) \geq 1\right\}$.

Case 2 : If $r>2$ then $C_{x_{0}, r}^{S}=\varnothing$.

Case 3 : If $r<2$ then $C_{x_{0}, r}^{S}=C_{x_{0}, \frac{r}{2}}$, where $C_{x_{0}, \frac{r}{2}}=\left\{x \in X: d\left(x, x_{0}\right)=\frac{r}{2}\right\}$.

Example 2.5. Let $X$ be a nonempty set, the function $d: X^{2} \rightarrow[0, \infty)$ be any metric on $X$ and the $S$-metric space be defined as in Example 2.3. Let us consider the circle $C_{x_{0}, r}^{S}$ according to the $S$-metric:

$$
C_{x_{0}, r}^{S}=\left\{x \in X: \mathcal{S}\left(x, x, x_{0}\right)=\min \left\{1, d\left(x, x_{0}\right)\right\}+\left|x-x_{0}\right|=r\right\} .
$$

Then we have the following cases:

Case 1 : If $x \in\left(X \backslash D_{x_{0}, 1}\right) \cup C_{x_{0}, 1}$ then $C_{x_{0}, r}^{S}=\left\{x \in\left(X \backslash D_{x_{0}, 1}\right) \cup C_{x_{0}, 1}:\left|x-x_{0}\right|=r-1\right\}$.

Case 2 : If $x \in D_{x_{0}, 1} \backslash C_{x_{0}, 1}$ then $C_{x_{0}, r}^{S}=\left\{x \in D_{x_{0}, 1} \backslash C_{x_{0}, 1}: d\left(x, x_{0}\right)+\left|x-x_{0}\right|=r\right\}$.

In the following example, the $S$-metric is not generated by any metric but any circle on this $S$-metric space is the same as the circle on the usual metric space $\mathbb{R}$ (or C).

Example 2.6. Let $X=\mathbb{R}\left(\right.$ or $\mathbb{C}$ ) and the function $\mathcal{S}: X^{3} \rightarrow[0, \infty)$ be defined by

$$
\mathcal{S}(x, y, z)=\max \{|x-y|,|y-z|,|z-x|\},
$$

for all $x, y, z \in X$. Then the function $\mathcal{S}: X^{3} \rightarrow[0, \infty)$ is an $S$-metric on $X$ which is not generated by any metric. For any circle $C_{x_{0}, r}^{S}$ on this $S$-metric space we have $C_{x_{0}, r}^{S}=$ $\left\{x_{0}-r, x_{0}+r\right\}$ which is correspond to the circle $C_{x_{0}, r}$ with the equation $\left|y-x_{0}\right|=r$ on the usual metric space $\mathbb{R}$.

\section{Fixed-Circle Theorems via New Types of $F_{c}^{S}$-contractions}

In this section, we give new fixed-circle theorems using new types of the notion of an $F_{c}^{S}$-contraction introduced in [10]. At first, we recall the definition of a fixedcircle and the following family of functions which was introduced by Wardowski in [28].

Definition 3.1. [16] Let $(X, \mathcal{S})$ be an $S$-metric space, $C_{x_{0}, r}^{S}$ be a circle on $X$ and $T: X \rightarrow X$ be a self-mapping. If $T x=x$ for every $x \in C_{x_{0}, r}^{S}$ then the circle $C_{x_{0}, r}^{S}$ is called as the fixed circle of $T$.

Definition 3.2. $\quad[28]$ Let $\mathbb{F}$ be the family of all functions $F:(0, \infty) \rightarrow \mathbb{R}$ such that

$(F 1) F$ is strictly increasing,

$(F 2)$ For each sequence $\left\{\alpha_{n}\right\}$ in $(0, \infty)$ the following holds $\lim \alpha_{n}=0$ if and only if $\lim F\left(\alpha_{n}\right)=-\infty$,

(F3) There exists $k \in(0,1)$ such that $\lim _{\alpha \rightarrow 0^{+}} \alpha^{k} F(\alpha)=0$. 
Some functions that satisfy the conditions $(F 1),(F 2)$ and $(F 3)$ of Definition 3.2 are given in the following example (see [28] for more details).

Example 3.1. [28] The following functions defined by

$$
\begin{gathered}
F_{1}:(0, \infty) \rightarrow \mathbb{R}, F_{1}(x)=\ln (x), \\
F_{2}:(0, \infty) \rightarrow \mathbb{R}, F_{2}(x)=\ln (x)+x, \\
F_{3}:(0, \infty) \rightarrow \mathbb{R}, F_{3}(x)=-\frac{1}{\sqrt{x}}
\end{gathered}
$$

and

$$
F_{4}:(0, \infty) \rightarrow \mathbb{R}, F_{4}(x)=\ln \left(x^{2}+x\right)
$$

are the examples of Definition 3.2.

Using this family of functions, in [4], some new fixed-point theorems was obtained on $S$-metric spaces. In [10], it was introduced the following new contraction type to obtain some fixed-circle results on an $S$-metric space.

Definition 3.3. [10] Let $(X, \mathcal{S})$ be an $S$-metric space. A self-mapping $T$ on $X$ is said to be an $F_{c}^{S}$-contraction if there exist $F \in \mathbb{F}, t>0$ and $x_{0} \in X$ such that for all $x \in X$ the following holds:

$$
\mathcal{S}(T x, T x, x)>0 \Longrightarrow t+F(\mathcal{S}(T x, T x, x)) \leq F\left(\mathcal{S}\left(x, x, x_{0}\right)\right)
$$

In [24], Suzuki-Berinde type $F_{c}^{S}$-contractions were introduced for the same purpose. Now we define new types of $F_{c}^{S}$-contractions to get new fixed-circle results. To do this, we use some classical contraction conditions such as Ćirić-type, modified Hardy-Rogers type and Khan-type contractive conditions.

Let $(X, \mathcal{S})$ be an $S$-metric space and $T$ be a self-mapping on $X$. We will use the number $r$ defined by

$$
r=\inf \{\mathcal{S}(T x, T x, x): x \in X, x \neq T x\},
$$

in all of our results.

\section{1. Ćirić type fixed-circle results on $S$-metric spaces}

At first, we introduce the following Ćirić type $F_{c}^{S}$-contraction.

Definition 3.4. Let $(X, \mathcal{S})$ be an $S$-metric space and $T$ be a self-mapping on $X$. If there exist $F \in \mathbb{F}, t>0$ and $x_{0} \in X$ such that for all $x \in X$ the following holds:

$$
\mathcal{S}(T x, T x, x)>0 \Longrightarrow t+F(\mathcal{S}(T x, T x, x)) \leq F\left(m\left(x, x, x_{0}\right)\right),
$$


where

$$
m(x, x, y)=\max \left\{\begin{array}{c}
\mathcal{S}(x, x, y), \mathcal{S}(x, x, T x), \mathcal{S}(y, y, T y), \\
\frac{1}{2}[\mathcal{S}(x, x, T y)+\mathcal{S}(y, y, T x)]
\end{array}\right\}
$$

then the self-mapping $T$ is called a Ćirić type $F_{c}^{S}$-contraction on $X$.

An immediate consequence of this definition is the following proposition.

Proposition 3.1. Let $(X, \mathcal{S})$ be an $S$-metric space. If a self-mapping $T$ on $X$ is a Cirić-type $F_{c}^{S}$-contraction with $x_{0} \in X$ then we have $T x_{0}=x_{0}$.

Proof. Assume that $T x_{0} \neq x_{0}$. From the definition of a Ćirić-type $F_{c}^{S}$-contraction and Lemma 2.1, we get

$$
\begin{aligned}
\mathcal{S}\left(T x_{0}, T x_{0}, x_{0}\right) & >0 \Longrightarrow t+F\left[\mathcal{S}\left(T x_{0}, T x_{0}, x_{0}\right)\right] \leq F\left(m\left(x_{0}, x_{0}, x_{0}\right)\right) \\
& =F\left(\max \left\{\begin{array}{c}
\mathcal{S}\left(x_{0}, x_{0}, x_{0}\right), \mathcal{S}\left(x_{0}, x_{0}, T x_{0}\right), \mathcal{S}\left(x_{0}, x_{0}, T x_{0}\right), \\
\frac{1}{2}\left[\mathcal{S}\left(x_{0}, x_{0}, T x_{0}\right)+\mathcal{S}\left(x_{0}, x_{0}, T x_{0}\right)\right]
\end{array}\right\}\right) \\
& =F\left(\mathcal{S}\left(x_{0}, x_{0}, T x_{0}\right)\right) .
\end{aligned}
$$

This is a contradiction by the fact that $t>0$. Then we have $T x_{0}=x_{0}$.

Using Cirić type $F_{c}^{S}$-contractions, we give the following fixed-circle theorem.

Theorem 3.1. Let $(X, \mathcal{S})$ be an $S$-metric space, $T$ be a Ćirić type $F_{c}^{S}$-contractive self-mapping with $x_{0} \in X$ and $r$ be defined as in (3.1). If $\mathcal{S}\left(T x, T x, x_{0}\right)=r$ for all $x \in C_{x_{0}, r}^{S}$ then the circle $C_{x_{0}, r}^{S}$ is a fixed circle of $T$. In particular, $T$ fixes every circle $C_{x_{0}, \rho}^{S}$ where $\rho<r$ if $\mathcal{S}\left(T x, T x, x_{0}\right)=\rho$ for all $x \in C_{x_{0}, \rho}^{S}$.

Proof. Since $\mathcal{S}\left(T x, T x, x_{0}\right)=r$, the self-mapping $T$ maps $C_{x_{0}, r}^{S}$ into (or onto) itself. Let $x \in C_{x_{0}, r}^{S}$ be an arbitrary point. If $T x \neq x$, by the definition of $r$ we have $\mathcal{S}(T x, T x, x) \geq r$. Hence, using the Ćirić-type $F_{c}^{S}$-contractive property, Lemma 2.1, Proposition 3.1 and the fact that $F$ is increasing, we get

$$
\begin{aligned}
F(r) & \leq F(\mathcal{S}(T x, T x, x)) \leq F\left(m\left(x, x, x_{0}\right)\right)-t<F\left(m\left(x, x, x_{0}\right)\right) \\
& =F\left(\max \left\{\begin{array}{c}
\mathcal{S}\left(x, x, x_{0}\right), \mathcal{S}(x, x, T x), \mathcal{S}\left(x_{0}, x_{0}, T x_{0}\right), \\
\frac{1}{2}\left[\mathcal{S}\left(x, x, T x_{0}\right)+\mathcal{S}\left(x_{0}, x_{0}, T x\right)\right]
\end{array}\right\}\right) \\
& =F(\max \{r, \mathcal{S}(x, x, T x), 0, r\})=F(\mathcal{S}(T x, T x, x)),
\end{aligned}
$$

which is a contradiction. Therefore, $\mathcal{S}(T x, T x, x)=0$ and so $T x=x$. Consequently, $C_{x_{0}, r}^{S}$ is a fixed circle of $T$.

Using the similar arguments, it is easy to see that $T$ also fixes any circle $C_{x_{0}, \rho}^{S}$ where $\rho<r$. 
Remark 3.1. 1) Notice that, in Theorem 3.1, Ćirić type $F_{c}^{S}$-contractive self-mapping $T$ fixes the disc $D_{x_{0}, r}^{S}$ if $\mathcal{S}\left(T x, T x, x_{0}\right)=\rho$ for all $x \in C_{x_{0}, \rho}^{S}$ and each $\rho \leq r$.

2) In Theorem 3.1, if $r=0$, then we have $C_{x_{0}, r}^{S}=\left\{x_{0}\right\}$ and this is a fixed circle of the self-mapping $T$ by Proposition 3.1 .

In the following example, we see that the converse statement of Theorem 3.1 is not always true.

Example 3.2. Let $X=\mathbb{C}$ be the $S$-metric space with the usual $S$-metric defined in Example 2.1, $z_{0} \in \mathbb{C}$ be any point and the self-mapping $T: X \rightarrow X$ be defined as

$$
T z=\left\{\begin{array}{cl}
z & , \quad\left|z-z_{0}\right| \leq \frac{\mu}{2} \\
z_{0} & , \quad\left|z-z_{0}\right|>\frac{\mu}{2}
\end{array},\right.
$$

for all $z \in \mathbb{C}$ with $\mu>0$. We show that $T$ is not a Ćirić-type $F_{c}^{S}$-contractive self-mapping. Indeed, if $\left|z-z_{0}\right|>\frac{\mu}{2}$ for $z \in \mathbb{C}$, then using Lemma 2.1 and the Ćirić-type $F_{c}^{S}$-contractive property, we get

$$
\begin{gathered}
\mathcal{S}(T z, T z, z)=\mathcal{S}\left(z_{0}, z_{0}, z\right)>0 \Longrightarrow t+F\left(\mathcal{S}\left(z_{0}, z_{0}, z\right)\right) \leq F\left(m\left(z, z, z_{0}\right)\right), \\
t+F\left(\mathcal{S}\left(z_{0}, z_{0}, z\right)\right) \leq F\left(\mathcal{S}\left(z, z, z_{0}\right)\right)
\end{gathered}
$$

and so

$$
t+F(r) \leq F(r) \Longrightarrow t \leq 0
$$

This is a contradiction since $t>0$. Hence $T$ is not a Ćirić-type $F_{c}^{S}$-contractive self-mapping for any $z_{0} \in \mathbb{C}$. But $T$ fixes every circle $C_{x_{0}, \rho}^{S}$ where $\rho \leq \mu$.

Now we give some illustrative examples of Theorem 3.1.

Example 3.3. Let $X=\{z \in \mathbb{C}:|z|=2\}$. Let us consider the $S$-metric $\mathcal{S}$ defined in Example 2.6 on $X$ and define the self-mapping $T: X \rightarrow X$ by

$$
T z=\left\{\begin{array}{ccc}
-2 & , & \frac{\pi}{3} \leq \arg (z) \leq \frac{\pi}{2} \\
z & , & \text { otherwise }
\end{array} .\right.
$$

Then the self-mapping $T$ is a Ćirić-type $F_{c}^{S}$-contractive self-mapping with $F=\ln x, t=$ $\ln \left(\frac{\sqrt{8+4 \sqrt{3}}}{2 \sqrt{3}}\right)$ and $z_{0}=-2 i$. Indeed, we obtain

$$
\begin{aligned}
r & =\inf \{\mathcal{S}(z, z, T z): z \in X, z \neq T z\} \\
& =2 \sqrt{2}
\end{aligned}
$$

In the case $\mathcal{S}(z, z, T z)>0$, we find

$$
\begin{aligned}
m(z, z,-2 i) & =\max \left\{\begin{array}{c}
\mathcal{S}(z, z,-2 i), \mathcal{S}(z, z,-2), \mathcal{S}(-2 i,-2 i,-2 i), \\
\frac{1}{2}[\mathcal{S}(z, z,-2 i)+\mathcal{S}(-2 i,-2 i,-2)]
\end{array}\right\} \\
& =\max \left\{|z+2 i|,|z+2|, 0, \frac{1}{2}[|z+2 i|+|2 i-2|]\right\} \\
& =\sqrt{8+4 \sqrt{3}}
\end{aligned}
$$


and hence

$$
t+\ln (|z+2|) \leq \ln (\sqrt{8+4 \sqrt{3}}) .
$$

Clearly, $T$ fixes the circle $C_{-2 i, 2 \sqrt{2}}^{S}=\{-2,2\}$ and the disc $D_{-2 i, 2 \sqrt{2}}^{S}=\{z \in X: \mathcal{S}(z, z,-2 i) \leq 2 \sqrt{2}\}$.

\subsection{Modified Hardy-Rogers type fixed-circle results on $S$-metric spaces}

Now we introduce the following modified Hardy-Rogers type $F_{c}^{S}$-contraction.

Definition 3.5. Let $(X, \mathcal{S})$ be an $S$-metric space and $T$ be a self-mapping on $X$. If there exist $F \in \mathbb{F}, t>0$ and $x_{0} \in X$ such that for all $x \in X$ the following holds

$$
\begin{aligned}
\mathcal{S}(T x, T x, x)> & 0 \Longrightarrow t+F(\mathcal{S}(T x, T x, x)) \leq \\
& F\left[\begin{array}{c}
\alpha \mathcal{S}\left(x, x, x_{0}\right)+\beta \mathcal{S}\left(T x_{0}, T x_{0}, x\right)+\gamma \mathcal{S}\left(T x, T x, x_{0}\right) \\
+\eta \frac{\mathcal{S}\left(T x_{0}, T x_{0}, x_{0}\right)[1+\mathcal{S}(T x, T x, x)]}{\left[1+\mathcal{S}\left(T x_{0}, T x_{0}, x\right)\right]}+\lambda\left(T x_{0}, T x_{0}, x_{0}\right)+\mathcal{S}\left(T x, T x, x_{0}\right) \\
+\mu \frac{\mathcal{S}(T x, T x, x)\left[1+\mathcal{S}\left(T x, T\left(T, x_{0}, T x_{0}, x_{0}\right) \cdot \mathcal{S}\left(x_{0}, x_{0}, x\right)\right.\right.}{1+\mathcal{S}\left(x, x, x_{0}\right)+\mathcal{S}\left(T x_{0}, T x_{0}, x_{0}\right)}
\end{array}\right],
\end{aligned}
$$

where $\alpha+\beta+\gamma+\eta+\lambda+\mu<\frac{1}{2}, \alpha, \beta, \gamma, \eta, \lambda, \mu \geq 0$ and $a \neq 0$, then the self-mapping $T$ is called a modified Hardy-Rogers type $F_{c}^{S}$-contraction on $X$.

Proposition 3.2. Let $(X, \mathcal{S})$ be an $S$-metric space. If a self-mapping $T$ on $X$ is a modified Hardy-Rogers type $F_{c}^{S}$-contraction with $x_{0} \in X$ then we have $T x_{0}=x_{0}$.

Proof. Assume that $T x_{0} \neq x_{0}$. By the hypothesis, we obtain

$$
\begin{aligned}
\mathcal{S}\left(T x_{0}, T x_{0}, x_{0}\right)> & 0 \Longrightarrow t+F\left(\mathcal{S}\left(T x_{0}, T x_{0}, x_{0}\right)\right) \leq \\
& F\left[\begin{array}{c}
\alpha \mathcal{S}\left(x_{0}, x_{0}, x_{0}\right)+\beta \mathcal{S}\left(T x_{0}, T x_{0}, x_{0}\right)+\gamma \mathcal{S}\left(T x_{0}, T x_{0}, x_{0}\right) \\
+\eta \frac{\mathcal{S}\left(T x_{0}, T x_{0}, x_{0}\right)\left[1+\mathcal{S}\left(T x_{0}, T x_{0}, x_{0}\right)\right]}{\left[1+\mathcal{S}\left(T x_{0}, T x_{0}, x_{0}\right)\right]}+\lambda \frac{\mathcal{S}\left(T x_{0}, T x_{0}, x_{0}\right)+\mathcal{S}\left(T x_{0}, T x_{0}, x_{0}\right)}{1+\mathcal{S}\left(T x_{0}, T x_{0}, x_{0}\right) \cdot \mathcal{S}\left(x_{0}, x_{0}, x_{0}\right)} \\
+\mu \frac{\mathcal{S}\left(T x_{0}, T x_{0}, x_{0}\right)\left[1+\mathcal{S}\left(T x_{0}, T x_{0}, x_{0}\right)\right]}{1+\mathcal{S}\left(x_{0}, x_{0}, x_{0}\right)+\mathcal{S}\left(T x_{0}, T x_{0}, x_{0}\right)}
\end{array}\right] \\
= & F\left[(\beta+\gamma+\eta+2 \lambda+\mu) \mathcal{S}\left(T x_{0}, T x_{0}, x_{0}\right)\right] \\
< & F\left[\mathcal{S}\left(T x_{0}, T x_{0}, x_{0}\right)\right] .
\end{aligned}
$$

This is a contradiction since $t>0$. Hence we get $T x_{0}=x_{0}$.

Now using the notion of a modified Hardy-Rogers type $F_{c}^{S}$-contraction condition, we prove the following fixed-circle theorem.

Theorem 3.2. Let $(X, \mathcal{S})$ be an $S$-metric space, $T$ be a modified Hardy-Rogers type $F_{c}^{S}$-contractive self-mapping with $x_{0} \in X$ and $r$ be defined as in (3.1). If $\mathcal{S}\left(T x, T x, x_{0}\right)=r$ for all $x \in C_{x_{0}, r}^{S}$ then $C_{x_{0}, r}^{S}$ is a fixed circle of $T$. In particular, $T$ fixes every circle $C_{x_{0}, \rho}^{S}$ where $\rho<r$ if $\mathcal{S}\left(T x, T x, x_{0}\right)=\rho$ for all $x \in C_{x_{0}, \rho}^{S}$. 
Proof. Let $x \in C_{x_{0}, r}^{S}$ and $T x \neq x$. If $r=0$, then we have $C_{x_{0}, r}^{S}=\left\{x_{0}\right\}$ and this is a fixed circle of the self-mapping $T$ by Proposition 3.2. Assume that $r>0$. Using the modified Hardy-Rogers type $F_{c}^{S}$-contraction property, Proposition 3.2, Lemma 2.1 and the fact that $F$ is increasing, we get

$$
\begin{aligned}
F(r) & \leq F(\mathcal{S}(T x, T x, x)) \\
& \leq F\left[\begin{array}{c}
\alpha \mathcal{S}\left(x, x, x_{0}\right)+\beta \mathcal{S}\left(T x_{0}, T x_{0}, x\right)+\gamma \mathcal{S}\left(T x, T x, x_{0}\right) \\
+\eta \frac{\mathcal{S}\left(T x_{0}, T x_{0}, x_{0}\right)[1+\mathcal{S}(T x, T x, x)]}{\left[1+\mathcal{S}\left(T x_{0}, T x_{0}, x\right)\right]}+\lambda \frac{\mathcal{S}\left(T x_{0}, T x_{0}, x_{0}\right)+\mathcal{S}\left(T x, T x, x_{0}\right)}{1+\mathcal{S}\left(T x_{0}, T x_{0}, x_{0}\right) \cdot \mathcal{S}\left(x, x, x_{0}\right)} \\
+\mu \frac{\mathcal{S}(T x, T x, x)\left[1+\mathcal{S}\left(T x, T x, x_{0}\right)\right]}{1+\mathcal{S}\left(x, x, x_{0}\right)+\mathcal{S}\left(T x_{0}, T x_{0}, x_{0}\right)}
\end{array}\right]-t \\
& <F[\alpha r+\beta r+\gamma r+\lambda r+\mu \mathcal{S}(T x, T x, x)] \\
& \leq F[(\alpha+\beta+\gamma+\lambda+\mu) \mathcal{S}(T x, T x, x)] \\
& \leq F[\mathcal{S}(T x, T x, x)]
\end{aligned}
$$

which is a contradiction. Therefore, $\mathcal{S}(T x, T x, x)=0$ and so $T x=x$. Consequently, $C_{x_{0}, r}^{S}$ is a fixed circle of $T$. Using the similar arguments, it is easy to see that $T$ also fixes any circle $C_{x_{0}, \rho}^{S}$ where $\rho<r$.

Remark 3.2. 1) Let $(X, S)$ be an $S$-metric space, $T$ be a modified Hardy-Rogers type $F_{c}^{S}$-contractive self-mapping with $x_{0} \in X$ and $r$ be defined as in (3.1). If $\mathcal{S}\left(T x, T x, x_{0}\right)=\rho$ for all $x \in C_{x_{0}, \rho}^{S}$ and each $\rho \leq r$, then $T$ fixes the disc $D_{x_{0}, r}^{S}$.

2) Let us consider the self-mapping $T$ given in Example 3.2. Then it can be easily seen that $T$ is not a modified Hardy-Rogers type $F_{c}^{S}$-contractive self-mapping. But, $T$ fixes every circle $C_{x_{0}, \rho}^{S}$ where $\rho \leq r$. Hence the converse statement of Theorem 3.2 is not always true.

Example 3.4. Let $X=\mathbb{R}^{+}$and the $S$-metric $\mathcal{S}$ be the usual $S$-metric. Let us define the self-mapping $T: X \rightarrow X$ as

$$
T x=\left\{\begin{array}{ccc}
2 x+\frac{4}{x} & , & x \in[1,4) \\
x & , & \text { otherwise }
\end{array},\right.
$$

for all $x \in X$. Then the self-mapping $T$ is a modified Hardy-Rogers type $F_{c}^{S}$-contractive self-mapping with $\alpha=\frac{1}{4}, \beta=\frac{1}{25}, \gamma=\frac{1}{25}, \lambda=\frac{1}{25}, \mu=\frac{1}{25}, F=\ln x, t=\ln \frac{9}{8}$ and $x_{0}=35$. Indeed, in the cases $\mathcal{S}(T x, T x, x)>0$ we find

$$
8 \leq \mathcal{S}(T x, T x, x) \leq 10
$$

and

$$
62 \leq \mathcal{S}\left(x, x, x_{0}\right) \leq 68
$$

and hence

$$
\begin{aligned}
t+F\left(2\left|x+\frac{4}{x}\right|\right) & \leq F[2 \alpha|x-35|] \\
& \leq F\left[\begin{array}{c}
2 \alpha|x-35|+2 \beta|x-35|+2 \gamma|T x-35| \\
+\eta \cdot 0+2 \lambda|T x-35| \\
+\mu \frac{2\left|x+\frac{4}{x}\right|[1+|T x-35|]}{1+2|x-35|}
\end{array}\right] .
\end{aligned}
$$


Also we have

$$
r=\inf \{\mathcal{S}(T x, T x, x): x \neq T x\}=8 .
$$

Therefore, the self-mapping $T$ fixes the circle $C_{35,8}^{S}=\{31,39\}$ and the disc $D_{35,8}^{S}=$ $\left\{x \in \mathbb{R}^{+}: 31 \leq x \leq 39\right\}$.

\subsection{Khan-type fixed-circle results on $S$-metric spaces}

Now we introduce the following Khan-type $F_{c}^{S}$-contraction.

Definition 3.6. Let $(X, \mathcal{S})$ be an $S$-metric space and $T$ be a self-mapping on $X$. If there exist $F \in \mathbb{F}, t>0$ and $x_{0} \in X$ such that for all $x \in X$ the following holds:

$$
\begin{aligned}
\mathcal{S}(T x, T x, x) & >0 \Longrightarrow t+F(\mathcal{S}(T x, T x, x)) \\
& \leq F\left[h \frac{\mathcal{S}(T x, T x, x) \mathcal{S}\left(T x_{0}, T x_{0}, x\right)+\mathcal{S}\left(T x_{0}, T x_{0}, x\right) \mathcal{S}\left(T x, T x, x_{0}\right)}{\mathcal{S}\left(T x_{0}, T x_{0}, x\right)+\mathcal{S}\left(T x, T x, x_{0}\right)}\right],
\end{aligned}
$$

where

$$
h \in[0,1), \mathcal{S}\left(T x_{0}, T x_{0}, x\right)+\mathcal{S}\left(T x, T x, x_{0}\right) \neq 0 .
$$

Then the self-mapping $T$ is called Khan-type $F_{c}^{S}$-contraction on $X$.

Proposition 3.3. Let $(X, \mathcal{S})$ be an $S$-metric space. If a self-mapping $T$ on $X$ is a Khan-type $F_{C}^{S}$-contraction with $x_{0} \in X$. Then we have $T x_{0}=x_{0}$.

Proof. Assume that $T x_{0} \neq x_{0}$. By the hypothesis, we have

$$
\begin{aligned}
\mathcal{S}\left(T x_{0}, T x_{0}, x_{0}\right) & >0 \Longrightarrow t+F\left(\mathcal{S}\left(T x_{0}, T x_{0}, x_{0}\right)\right) \\
& \leq F\left[h \frac{\mathcal{S}\left(T x_{0}, T x_{0}, x_{0}\right) \mathcal{S}\left(T x_{0}, T x_{0}, x_{0}\right)+\mathcal{S}\left(T x_{0}, T x_{0}, x_{0}\right) \mathcal{S}\left(T x_{0}, T x_{0}, x_{0}\right)}{\mathcal{S}\left(T x_{0}, T x_{0}, x_{0}\right)+\mathcal{S}\left(T x_{0}, T x_{0}, x_{0}\right)}\right] \\
& =F\left[h \frac{\mathcal{S}^{2}\left(T x_{0}, T x_{0}, x_{0}\right)+\mathcal{S}^{2}\left(T x_{0}, T x_{0}, x_{0}\right)}{2 \mathcal{S}\left(T x_{0}, T x_{0}, x_{0}\right)}\right] \\
& =F\left[h \frac{2 \mathcal{S}^{2}\left(T x_{0}, T x_{0}, x_{0}\right)}{2 \mathcal{S}\left(T x_{0}, T x_{0}, x_{0}\right)}\right] \\
& <F\left[\mathcal{S}\left(T x_{0}, T x_{0}, x_{0}\right)\right]
\end{aligned}
$$

which is contradiction since $t>0$. Then we have $T x_{0}=x_{0}$.

Now using the notion of a Khan-type $F_{c}^{S}$-contraction condition, we prove the following fixed-circle theorem.

Theorem 3.3. Let $(X, \mathcal{S})$ be an $S$-metric space, $T$ be a Khan-type $F_{c}^{S}$-contraction with $x_{0} \in X$ and $r$ be defined as in (3.1). If $\mathcal{S}\left(T x, T x, x_{0}\right)=r$ for all $x \in C_{x_{0}, r}^{S}$ then $C_{x_{0}, r}^{S}$ is a fixed circle of $T$. In particular, $T$ fixes every circle $C_{x_{0}, \rho}^{S}$ with $\rho<r$ if $\mathcal{S}\left(T x, T x, x_{0}\right)=\rho$ for all $x \in C_{x_{0}, \rho}^{S}$. 
Proof. Let $x \in C_{x_{0}, r}^{S}$ and $T x \neq x$. If $r=0$, then we have $C_{x_{0}, r}^{S}=\left\{x_{0}\right\}$ and this is a fixed circle of the self-mapping $T$ by Proposition 3.3.

Assume that $r>0$. Using the Khan-type $F_{C}^{S}$-contractive property, Proposition 3.3, Lemma 2.1 and the fact that $F$ is increasing, we get

$$
\begin{aligned}
F(r) & \leq F(\mathcal{S}(T x, T x, x)) \\
& \leq F\left[h \frac{\mathcal{S}(T x, T x, x) \mathcal{S}\left(T x_{0}, T x_{0}, x\right)+\mathcal{S}\left(T x_{0}, T x_{0}, x\right) \mathcal{S}\left(T x, T x, x_{0}\right)}{\mathcal{S}\left(T x_{0}, T x_{0}, x\right)+\mathcal{S}\left(T x, T x, x_{0}\right)}\right]-t \\
& <F\left[h \frac{\mathcal{S}(T x, T x, x) r+r^{2}}{2 r}\right]=F\left[h \frac{\mathcal{S}(T x, T x, x)+r}{2}\right] \\
& \leq F\left[h \frac{\mathcal{S}(T x, T x, x)+\mathcal{S}(T x, T x, x)}{2}\right]=F[h \mathcal{S}(T x, T x, x)] \\
& <F[\mathcal{S}(T x, T x, x)]
\end{aligned}
$$

which is a contradiction. Therefore we have $\mathcal{S}(T x, T x, x)=0$ and so $T x=x$. Consequently, $C_{x_{0}, r}^{S}$ is a fixed circle of $T$.

By the similar arguments, it is easy to verify that $T$ also fixes any circle $C_{x_{0}, \rho}^{S}$ where $\rho<r$.

Remark 3.3. Notice that, in Theorem 3.3, Khan-type $F_{c}^{S}$-contractive self-mapping $T$ fixes the disc $D_{x_{0}, r}^{S}$ if $\mathcal{S}\left(T x, T x, x_{0}\right)=\rho$ for all $x \in C_{x_{0}, \rho}^{S}$ and each $\rho \leq r$. Therefore, the center of any fixed circle is also fixed by $T$.

Now we give the following illustrative example.

Example 3.5. Let $X=\left\{e^{k}: k \in \mathbb{N}\right\}$ and the $S$-metric be defined as in [14] such that

$$
\mathcal{S}(x, y, z)=\left|\ln \frac{x}{y}\right|+\left|\ln \frac{x y}{z^{2}}\right|
$$

for all $x, y, z \in X$ (see Example 2.6 on page 12 in [14]). Let us define the self-mapping $T: X \rightarrow X$ as

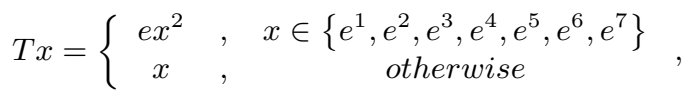

for all $x \in X$. Then the self-mapping $T$ is a Khan-type $F_{c}^{S}$-contractive self-mapping with $F=-\frac{1}{\sqrt{x}}, t=\frac{1}{8}-\frac{1}{4 \sqrt{5}}$ and $x_{0}=e^{23}$. Indeed, in the case $\mathcal{S}(T x, T x, x)>0$, we find

$$
\mathcal{S}(T x, T x, x) \in\{4,6,8,10,12,14,16\}
$$

and

$$
20<h \frac{\mathcal{S}(T x, T x, x) \mathcal{S}\left(T x_{0}, T x_{0}, x\right)+\mathcal{S}\left(T x_{0}, T x_{0}, x\right) \mathcal{S}\left(T x, T x, x_{0}\right)}{\mathcal{S}\left(T x_{0}, T x_{0}, x\right)+\mathcal{S}\left(T x, T x, x_{0}\right)},
$$

where $h=\frac{20}{21}$. Then we have

$t+F(\mathcal{S}(T x, T x, x)) \leq F\left[h \frac{\mathcal{S}(T x, T x, x) \mathcal{S}\left(T x_{0}, T x_{0}, x\right)+\mathcal{S}\left(T x_{0}, T x_{0}, x\right) \mathcal{S}\left(T x, T x, x_{0}\right)}{\mathcal{S}\left(T x_{0}, T x_{0}, x\right)+\mathcal{S}\left(T x, T x, x_{0}\right)}\right]$ 
We obtain

$$
r=\inf \{\mathcal{S}(T x, T x, x): x \neq T x\}=4
$$

and therefore, the self-mapping $T$ fixes the circle $C_{e^{23}, 4}^{S}=\left\{e^{21}, e^{25}\right\}$ and the $\operatorname{disc} D_{e^{23}, 4}^{S}=$ $\left\{e^{21}, e^{22}, e^{23}, e^{24}, e^{25}\right\}$.

\section{Fixed-Circle Theorems via Auxiliary Functions}

In this section, we investigate the existence and uniqueness theorems for fixed circles of self-mappings using some auxiliary functions. Let $r>0$ be any real number. We consider the function $\varphi_{r}: \mathbb{R}^{+} \cup\{0\} \rightarrow \mathbb{R}$ defined as

$$
\varphi_{r}(u)=\left\{\begin{array}{ccc}
u-r & , & u>0 \\
0 & , & u=0
\end{array},\right.
$$

for all $u \in \mathbb{R}^{+} \cup\{0\}$ [12]. Using the function $\varphi_{r}$ we give the following theorem.

Theorem 4.1. Let $(X, \mathcal{S})$ be an $S$-metric space and $C_{x_{0}, r}^{S}$ be any circle on $X$. Consider the function $\varphi_{r}$ defined in (4.1). If there exists a self-mapping $T: X \rightarrow X$ satisfying the conditions

1. $\mathcal{S}\left(T x, T x, x_{0}\right)=r$ for each $x \in C_{x_{0}, r}^{S}$,

2. $\mathcal{S}(T x, T x, T y)>r$ for each $x, y \in C_{x_{0}, r}^{S}$ and $x \neq y$,

3. $\mathcal{S}(T x, T x, T y) \leq \mathcal{S}(x, x, y)-\varphi_{r}(\mathcal{S}(x, x, T x))$ for each $x, y \in C_{x_{0}, r}^{S}$,

then the circle $C_{x_{0}, r}^{S}$ is a fixed circle of $T$.

Proof. Let $x \in C_{x_{0}, r}^{S}$ be an arbitrary point. By the condition (1), we have $T x \in$ $C_{x_{0}, r}^{S}$ for all $x \in C_{x_{0}, r}^{S}$. Now we prove that $x$ is a fixed point of $T$. On the contrary, let us assume that $T x \neq x$. Taking $y=T x$ and using the condition (2), we find

$$
\mathcal{S}\left(T x, T x, T^{2} x\right)>r .
$$

Using the condition (3), we have

$$
\begin{aligned}
\mathcal{S}\left(T x, T x, T^{2} x\right) & \leq \mathcal{S}(x, x, T x)-\varphi_{r}(\mathcal{S}(x, x, T x)) \\
& =\mathcal{S}(x, x, T x)-\mathcal{S}(x, x, T x)+r=r .
\end{aligned}
$$

Combining the inequalities (4.2) and (4.3), we get a contradiction. Hence it should be $T x=x$. Consequently, the circle $C_{x_{0}, r}^{S}$ is a fixed circle of $T$. 
Remark 4.1. Notice that the condition (1) in Theorem 4.1 guarantees that $T x$ is on the circle $C_{x_{0}, r}^{S}$ for $x \in C_{x_{0}, r}^{S}$, the condition (2) shows that the distance of the images of any two elements on the circle $C_{x_{0}, r}^{S}$ can not be less than (or equal to) $r$.

Now we give an example of a self-mapping which has a fixed-circle on an $S$-metric space.

Example 4.1. Let $X=\mathbb{R}$ and the metric function $d: X^{2} \rightarrow[0, \infty)$ be defined by

$$
d(x, y)=\left\{\begin{array}{cc}
0 & , \quad x=y \\
|x|+|y| & , \quad x \neq y
\end{array},\right.
$$

for all $x, y \in X$. Let us consider the $S$-metric defined in Example 2.2. The circle $C_{\frac{1}{2}, 1}^{S}=$ $\left\{x \in X: \mathcal{S}\left(x, x, \frac{1}{2}\right)=1\right\}=\{0\}$. If we consider the self-mapping $T_{1}: \mathbb{R} \rightarrow \mathbb{R}$ defined by

$$
T_{1} x=\left\{\begin{array}{ll}
4 & , \quad x=\frac{1}{2} \\
0 & , \quad x \neq \frac{1}{2}
\end{array},\right.
$$

for all $x \in \mathbb{R}$ then the self-mapping $T_{4}$ satisfies the conditions of Theorem 4.1 and $T_{4}$ fixes the circle $C_{\frac{1}{2}, 1}^{S}$.

In the following example, we see that the converse statement of Theorem 4.1 is not always true.

Example 4.2. Let $X=\mathbb{C}$ and consider the $S$-metric defined in Example 2.6. Let us consider the circle $C_{0, \frac{1}{3}}^{S}$ and define the self-mapping $T_{2}: \mathbb{C} \rightarrow \mathbb{C}$

$$
T_{2} z=\left\{\begin{array}{cc}
\frac{1}{9 \bar{z}} & , \quad z \neq 0 \\
0 & , \quad z=0
\end{array},\right.
$$

for all $z \in \mathbb{C}$, where $\bar{z}$ denotes the complex conjugate of the complex number $z$. Clearly, we have $T_{2}\left(C_{0, \frac{1}{3}}^{S}\right)=\left(C_{0, \frac{1}{3}}^{S}\right)$. It can be easily checked that the self mapping $T_{2}$ does not satisfy the condition (2) of Theorem 4.1. But, an easy computation shows that $T_{2}$ fixes the circle $C_{0, \frac{1}{3}}^{S}$.

In the following example we see that the circle need not to be fixed even if $T\left(C_{x_{0}, r}^{S}\right)=C_{x_{0}, r}^{S}$.

Example 4.3. Let $(\mathbb{C}, \mathcal{S})$ be the usual $S$-metric space. Let us consider the circle $C_{0, \frac{1}{8}}^{S}$ and define the self-mapping $T_{3}: \mathbb{C} \rightarrow \mathbb{C}$ as

$$
T_{3} z=\left\{\begin{array}{cc}
\frac{1}{16 z} \quad, \quad z \neq 0 \\
0 \quad, \quad z=0
\end{array}\right.
$$

for all $z \in \mathbb{C}$. Then we have $T_{3}\left(C_{0, \frac{1}{8}}^{S}\right)=C_{0, \frac{1}{8}}^{S}$. But the self-mapping $T_{3}$ does not satisfy the conditions (2) and (3) of Theorem 4.1. Clearly, the circle $C_{0, \frac{1}{8}}^{S}$ is not a fixed circle of $T_{3}$ since $T_{3}\left(\frac{i}{4}\right)=-\frac{i}{4}$ and $T_{3}\left(-\frac{i}{4}\right)=\frac{i}{4}$. More precisely, $T_{3}$ fixes only the points $\frac{1}{4}$ and $-\frac{1}{4}$ on the circle $C_{0, \frac{1}{8}}^{S}$. 
In the following example we see that a self mapping can be fix more than one circle.

Example 4.4. Let $X=\mathbb{R}$ and $(X, \mathcal{S})$ be the $S$-metric space defined in Example 2.6. Let us consider the circles $C_{0,4}^{S}$ and $C_{6,2}^{S}$ and the self-mapping $T_{4}: \mathbb{R} \rightarrow \mathbb{R}$ as

$$
T_{4} x=\left\{\begin{array}{ccc}
\frac{2 x+4}{x+5} & , & x \in(-\infty, 4) \\
\frac{17 x+56}{24} & , & x \in(4, \infty) \\
4 & , & x=4
\end{array},\right.
$$

for all $x \in \mathbb{R}$. It can be easily checked that the self-mapping $T_{4}$ satisfies the conditions of Theorem 4.1 and that both of the circles $C_{0,4}^{S}$ and $C_{6,2}^{S}$ are the fixed circles of $T_{4}$.

Now we give another existence theorem for fixed circles.

Theorem 4.2. Let $(X, \mathcal{S})$ be an $S$-metric space and $C_{x_{0}, r}^{S}$ be any circle on $X$. Let us define the mapping

$$
\varphi: X \rightarrow[0, \infty), \varphi(x)=\mathcal{S}\left(x, x, x_{0}\right)
$$

for all $x \in X$. If there exists a self-mapping $T: X \rightarrow X$ satisfying

1. $\mathcal{S}(x, x, T x) \leq \max \{\varphi(x), \varphi(T x)\}-r$,

2. $\mathcal{S}\left(T x, T x, x_{0}\right)-h \mathcal{S}(x, x, T x) \leq r$,

for all $x \in C_{x_{0}, r}^{S}$ and $h \in[0,1)$, then $C_{x_{0}, r}^{S}$ is a fixed circle of $T$.

Proof. Let $x \in C_{x_{0}, r}^{S}$. On the contrary, assume that $T x \neq x$. Then we have the following cases:

Case 1. If $\max \{\varphi(x), \varphi(T x)\}=\varphi(x)$ then using the condition (1) we have

$$
\mathcal{S}(x, x, T x) \leq \max \{\varphi(x), \varphi(T x)\}-r=\varphi(x)-r=r-r=0
$$

and so $\mathcal{S}(x, x, T x)=0$, a contradiction. Hence we get $T x=x$.

Case 2. If $\max \{\varphi(x), \varphi(T x)\}=\varphi(T x)$ then we obtain

$$
\mathcal{S}(x, x, T x) \leq \max \{\varphi(x), \varphi(T x)\}-r=\varphi(T x)-r
$$

and using the condition (2) we find

$$
\mathcal{S}(x, x, T x) \leq \varphi(T x)-r \leq h \mathcal{S}(x, x, T x)+r-r=h \mathcal{S}(x, x, T x),
$$

a contradiction since $h \in[0,1)$. Hence we get $T x=x$.

Consequently, $C_{x_{0}, r}^{S}$ is a fixed circle of $T$. 
Remark 4.2. (1) Notice that the condition (1) in Theorem 4.2 guarantees that $T x$ is not in the interior of the circle $C_{x_{0}, r}^{S}$ for $x \in C_{x_{0}, r}^{S}$. Similarly the condition (2) guarantees that $T x$ is not exterior of the circle $C_{x_{0}, r}^{S}$ for $x \in C_{x_{0}, r}^{S}$. Hence $T x \in C_{x_{0}, r}^{S}$ for each $x \in C_{x_{0}, r}^{S}$.

(2) Notice that the conditions of Theorem 4.2 are satisfied by the self-mapping $T_{2}$.

Now we give the following example.

Example 4.5. Let $X=\mathbb{R}$ be the $S$-metric space with the usual $S$-metric defined in Example 2.1. Let us consider the circle $C_{0,8}^{S}$ and define the self-mapping $T_{5}: \mathbb{R} \rightarrow \mathbb{R}$ as

$$
T_{5} x=\left\{\begin{array}{ccc}
2 & , & x \in\left\{-\frac{8}{\sqrt{3}}, 2\right\} \\
\frac{8 x+16 \sqrt{3}}{\sqrt{3} x+8} & , \quad x \in \mathbb{R} \backslash\left\{-\frac{8}{\sqrt{3}}, 2\right\}
\end{array},\right.
$$

for all $x \in \mathbb{R}$. Then the self-mapping $T_{5}$ satisfies the conditions (1) and (2) in Theorem 4.2. Hence $C_{0,8}^{S}$ is a fixed circle of $T_{5}$. Notice that $C_{3,2}^{S}$ is another fixed circle of $T_{5}$ and so the number of the fixed circles need not to be unique for a giving self-mapping.

Now, in the following example, we give an example of a self-mapping which satisfies the condition (1) and does not satisfy the condition (2) of Theorem 4.2.

Example 4.6. Let $X=\mathbb{R}$ and the $S$-metric be defined as in Example 2.6. Let us consider the circle $C_{0,6}^{S}$ and define the self-mapping $T_{6}: \mathbb{R} \rightarrow \mathbb{R}$ as

$$
T_{6} x=\left\{\begin{array}{ccc}
\frac{4 x+48 \sqrt{3}}{\sqrt{3} x+3} & , & x \in(-7,7) \\
20 & , & \text { otherwise }
\end{array},\right.
$$

for all $x \in \mathbb{R}$. Then the self-mapping $T_{6}$ satisfies the conditions (1) but does not satisfy the conditions (2) in Theorem 4.2. Consequently $C_{0,6}^{S}$ is not a fixed circle of $T_{6}$.

In the following, we give an example of a self-mapping which satisfies the condition (2) and does not satisfy the condition (1) in Theorem 4.2.

Example 4.7. Let $X=\mathbb{C}$ be the $S$-metric space with the usual $S$-metric defined in Example 2.1. Let us consider the circle $C_{0,12}^{S}$ and define the self-mapping $T_{7}: \mathbb{C} \rightarrow \mathbb{C}$ as

$$
T_{7} z=\left\{\begin{array}{cll}
\frac{\operatorname{Re}(z)}{2} & \text { if } & \operatorname{Re}(z) \geq 0 \\
-\frac{\operatorname{Re}(z)}{2} & \text { if } & \operatorname{Re}(z)<0
\end{array},\right.
$$

for all $z \in \mathbb{C}$. Then the self-mapping $T_{7}$ satisfies the condition (2) and does not satisfy the condition (1) in Theorem 4.2 .

Now we use the following corollaries to obtain a uniqueness theorem for fixed circles of self-mappings.

Corollary 4.1. [22] Let $(X, \mathcal{S})$ be a complete $S$-metric space and $T$ be a selfmapping of $X$, and

$$
S(T x, T x, T y) \leq a S(x, x, y)+b S(T x, T x, x)+c S(T y, T y, y),
$$

for some $a, b, c \geq 0, a+b+c<1$, and all $x, y \in X$. Then $T$ has a unique fixed point in $X$. Moreover, if $c<\frac{1}{2}$ then $T$ is continuous at the fixed point. 
Corollary 4.2. [22] Let $(X, \mathcal{S})$ be a complete $S$-metric space and $T$ be a selfmapping of $X$, and

$$
S(T x, T x, T y) \leq h \max \{S(T x, T x, y), S(T y, T y, x)\},
$$

for some $h \in\left[0, \frac{1}{3}\right)$ and all $x, y \in X$. Then $T$ has a unique fixed point in $X$. Moreover, $T$ is continuous at the fixed point.

We give the following theorem.

Theorem 4.3. Let $(X, \mathcal{S})$ be an $S$-metric space and $T: X \rightarrow X$ be a self-mapping with the fixed circle $C_{x_{0}, r}^{S}$. If one of the contractive conditions (4.4) or (4.5) is satisfied for all $x \in C_{x_{0}, r}^{S}, y \in X \backslash C_{x_{0}, r}^{S}$ by $T$ then $C_{x_{0}, r}^{S}$ is the unique fixed circle of $T$.

Proof. Assume that there exists two fixed circles $C_{x_{0}, r}^{S}$ and $C_{x_{0}, \rho}^{S}$ of the self-mapping $T$. Let $x \in C_{x_{0}, r}^{S}$ and $y \in C_{x_{0}, \rho}^{S}$ be arbitrary points with $x \neq y$. If the contractive condition (4.4) is satisfied by $T$, then we obtain

$$
\begin{aligned}
S(x, x, y) & =S(T x, T x, T y) \leq a S(x, x, y)+b S(T x, T x, x)+c S(T y, T y, y) \\
& =a S(x, x, y),
\end{aligned}
$$

which is a contradiction since $a+b+c<1$. Hence it should be $x=y$. Consequently $C_{x_{0}, r}^{S}$ is the unique fixed circle of $T$. Similarly, if the contractive condition (4.5) is satisfied by $T$ then we get

$S(x, x, y)=S(T x, T x, T y) \leq h \max \{S(T x, T x, y), S(T y, T y, x)\}=h S(x, x, y)$,

which is a contradiction since $h \in\left[0, \frac{1}{3}\right)$. Hence it should be $x=y$. Consequently $C_{x_{0}, r}^{S}$ is the unique fixed circle of $T$.

Now we consider the identity map $I_{X}: X \rightarrow X$ defined as $I_{X}(x)=x$ for all $x \in X$. We note that the identity map satisfies the conditions of Theorem 4.2 but can not satisfy the condition (2) of Theorem 4.1 everywhen. Therefore, we investigate a condition which excludes the identity map in Theorem 4.2 (resp. Theorem 4.1). For this purpose, we obtain the following theorem.

Theorem 4.4. Let $(X, \mathcal{S})$ be an $S$-metric space, $T: X \rightarrow X$ be a self mapping having a fixed circle $C_{x_{0}, r}^{S}$ and the mapping $\varphi_{r}$ be defined as in (4.1). The selfmapping $T: X \rightarrow X$ satisfies the condition

$$
\mathcal{S}(x, x, T x)<\varphi_{r}(\mathcal{S}(x, x, T x))+r,
$$

for all $x \in X$ if and only if $T=I_{X}$. 
Proof. Let $x \in X$ be any point and assume that $T x \neq x$. Then using the inequality (4.6), we get

$$
\mathcal{S}(x, x, T x)<\varphi_{r}(\mathcal{S}(x, x, T x))+r=\mathcal{S}(x, x, T x)-r+r,
$$

which is a contradiction. Hence we have $T x=x$ and $T=I_{X}$.

Conversely, it is clear that the identity map $I_{X}$ satisfies the condition (4.6).

\section{REFEREN CES}

1. H. Alolaiyan, B. Ali and M. AbBas: Characterization of a b-metric space completeness via the existence of a fixed point of Ciric-Suzuki type quasi-contractive multivalued operators and applications. An. Ştiinţ. Univ. "Ovidius" Constanţa Ser. Mat. 27(1) (2019), 5-33.

2. F. BoJor: Fixed points of Kannan mappings in metric spaces endowed with a graph. An. Ştiinţ. Univ. "Ovidius" Constanţa Ser. Mat. 20(1) (2012), 31-40.

3. LJ. B. CIRIC: A generalization of Banach's contraction principle. Proc. Amer. Math. Soc. 45 (1974), 267-273.

4. S. ChaipornjareansRi: Fixed point theorems for Fw-contractions in complete s-metric spaces. Thai J. Math. 14 (2016), Special issue, 98-109.

5. A. Fulga and E. KarAPINAR: Revisiting of some outstanding metric fixed point theorems via E-contraction. An. Ştiinţ. Univ. "Ovidius" Constanţa Ser. Mat. 26(3) (2018), 73-98.

6. A. Gupta: Cyclic contraction on S-metric space. Int. J. Anal. Appl. 3(2) (2013), $119-130$.

7. N. T. Hieu, N. T. Thanh Ly and N. V. Dung: A generalization of Ćirić quasicontractions for maps on S-metric spaces. Thai J. Math. 13(2) (2015), 369-380.

8. E. Karapinar, A. F. Roldán-López-De-Hierro and S. Bessem: Matkowski theorems in the context of quasi-metric spaces and consequences on G-metric spaces. An. Ştiinţ. Univ. "Ovidius" Constanţa Ser. Mat. 24(1) (2016), 309-333.

9. N. MlaikI: $\alpha-\psi$-contractive mapping on S-metric space. Math. Sci. Lett. 4(1) (2015), 9-12.

10. N. Mlaiki, U. Çelik, N. TaŞ, N. Y. ÖzGür and A. Mukheimer: Wardowski type contractions and the fixed-circle problem on S-metric spaces. J. Math. (2018), Art. ID 9127486, 9 pp.

11. N. Mlaiki, N. TAŞ and N. Y. ÖzGüR: On the fixed-circle problem and Khan type contractions. Axioms 7(4) (2018), 80.

12. N. Y. ÖZGür and N. TAŞ: Some fixed-circle theorems and discontinuity at fixed circle. In: AIP Conference Proceedings 1926(1), AIP Publishing LLC, 2018, pp. 020048 .

13. N. Y. ÖZgür and N. TAŞ: Some new contractive mappings on S-metric spaces and their relationships with the mapping (S25). Math. Sci. (Springer) 11(1) (2017), 7-16. 
14. N. Y. ÖZgür N. TAŞ and U. ÇELIK: New fixed-circle results on S-metric spaces. Bull. Math. Anal. Appl. 9(2) (2017), 10-23.

15. N. Y. ÖzGür and N. TAŞ: Some generalizations of fixed point theorems on $S$ metric spaces. Essays in Mathematics and Its Aplications in Honor of Vladimir Arnold, New York, Springer, 2016.

16. N. Y. ÖZGüR and N. TAŞ: Fixed-circle problem on S-metric spaces with a geometric viewpoint. Facta Univ. Ser. Math. Inform. 34(3) (2019), 459-472.

17. N. Y. ÖzGür and N. TAŞ: Some fixed-circle theorems on metric spaces. Bull. Malays. Math. Sci. Soc. 42(4) (2019), 1433-1449.

18. N. Y. ÖzGür and N. TAŞ: Some fixed point theorems on S-metric spaces. Mat. Vesnik 69(1) (2017), 39-52.

19. N. Y. ÖzGür and N. TAş: The Picard theorem on S-metric spaces. Acta Math. Sci. Ser. B (Engl. Ed.) 38(4) (2018), 1245-1258.

20. R. P. Pant, N. Y. Özgür and N. Taş: On Discontinuity Problem at Fixed Point. Bull. Malays. Math. Sci. Soc. 43(1) (2020), 499-517.

21. S. Sedghi, N. Shobe and A. Aliouche: A generalization of fixed point theorems in S-metric spaces. Mat. Vesnik 64(3) (2012), 258-266.

22. S. Sedghi and N. V. Dung: Fixed point theorems on S-metric spaces. Mat. Vesnik 66(1) (2014), 113-124.

23. N. Sounyah: A fixed point in partial $S_{b}$-metric spaces. An. Ştiinţ. Univ. "Ovidius" Constanţa Ser. Mat. 24(3) (2016), 351-362.

24. N. TAŞ: Suzuki-Berinde type fixed-point and fixed-circle results on S-metric spaces. J. Linear Topol. Algebra 7(3) (2018), 233-244.

25. N. TAŞ: Various types of fixed-point theorems on S-metric spaces. J. BAUN Inst. Sci. Technol. 20(2) (2018), 211-223.

26. N. TAŞ, N. Y. ÖzGÜr and N. Mlaiki: New types of $F_{C}$-contractions and the fixed-circle problem. Mathematics, 6(10) (2018), 188.

27. N. TAŞ and N. Y. ÖZGüR: Common fixed points of continuous mappings on S-metric spaces. Math. Notes. 104(3-4) (2018), 587-600.

28. D. WARDOWSKI: Fixed points of a new type of contractive mappings in complete metric spaces. Fixed Point Theory Appl. 2012, (2012):94, 6 pp.

Ufuk Çelik

Faculty of Arts and Sciences

Department of Mathematics

10145 Balıkesir, Turkey

ufuk. celik@baun.edu.tr

Nihal Özgür

Faculty of Arts and Sciences

Department of Mathematics

10145 Balıkesir, Turkey

nihal@balikesir.edu.tr 